\title{
A Rapid and Convenient Method for in Vivo Fluorescent Imaging of Protoscolices of Echinococcus multilocularis
}

\author{
Tao Yang', Sibo Wang', Xuyong Zhang', Jie Xia', Jun Guo', Jixue Hou', Hongwei Zhang1', Xueling Chen,", \\ Xiangwei $\mathrm{Wu}^{1,3, *}$ \\ 'Department of General Surgery, First Affiliated Hospital, Shihezi University School of Medicine, Shihezi, Xinjiang, China; '2Department of Immunology, \\ Shihezi University School of Medicine, Shihezi, Xinjiang, China; ${ }^{2}$ Laboratory of Transitional Medicine, Shihezi University School of Medicine, Shihezi, \\ Xinjiang, China
}

\begin{abstract}
Human and animal alveolar echinococcosis (AE) are important helminth infections endemic in wide areas of the Northern hemisphere. Monitoring Echinococcus multilocularis viability and spread using real-time fluorescent imaging in vivo provides a fast method to evaluate the load of parasite. Here, we generated a kind of fluorescent protoscolices in vivo imaging model and utilized this model to assess the activity against $E$. multilocularis protoscolices of metformin (Met). Results indicated that JC-1 tagged E. multilocularis can be reliably and confidently used to monitor protoscolices in vitro and in vivo. The availability of this transient in vivo fluorescent imaging of $E$. multilocularis protoscolices constitutes an important step toward the long term bio-imaging research of the AE-infected mouse models. In addition, this will be of great interest for further research on infection strategies and development of drugs and vaccines against $E$. multilocularis and other cestodes.
\end{abstract}

Key words: Echinococcus multilocularis, fluorescent imaging, in vivo, animal model

Alveolar echinococcosis (AE), which is considered to be one of the most lethal helminthic infections of humans, poses a great threat to millions of humans and animals in wide areas of the northern hemisphere $[1,2]$. The larval form of the foxtapeworm Echinococcus multilocularis is the causative agent of AE. Eggs which are accidentally ingested by the intermediate host are the source of infections in humans. The egg contains the infectious oncosphere, which actively penetrates the intestinal mucosa and migrates primarily to the liver via blood and lymphatic vessels. Then, later in the infection, it is possible to find blood metastasis to the lung, brain, bones, and local extension of the lesion (abdomen, retroperitoneum, and diaphragm) [3]. First symptoms are usually vague; patients may complain of fatigue, weight loss, or may have hepatomegaly. One third of them have cholestatic jaundice; one third present with abdominal pain. In advanced stages, liver failure usually occurs, and it is frequently associated with portal hypertension, ascites, and splenomegaly. The prognosis in untreated or

\footnotetext{
- Received 26 November 2015, revised 1 February 2016, accepted 1 February 2016.

*Corresponding author (wxwshz@126.com; xuelingch@hotmail.com)

(c) 2016, Korean Society for Parasitology and Tropical Medicine

This is an Open Access article distributed under the terms of the Creative Commons Attribution Non-Commercial License (http://creativecommons.org/licenses/by-nc/3.0) which permits unrestricted non-commercial use, distribution, and reproduction in any medium, provided the original work is properly cited.
}

inadequately treated patients with $\mathrm{AE}$ is poor.

Treatment of $\mathrm{AE}$ requires surgical intervention, if possible radical, combined with chemotherapy using benzimidazole carbamate derivatives, such as albendazole sulfoxide (ABZSO) and mebendazole sulfoxide (MBZSO). Chemotherapy has been shown to exert a parasitostatic rather than a parasiticidal effect [4]. A further disadvantage of the present treatment is that it has, in certain cases, proven to be ineffective, and the recurrence rate is rather high once chemotherapy is stopped [5]. Thus, the development of new treatments of AE is anticipated. Exploring novel molecules for use as antihydatid drugs or vaccines necessitates experimental animal models.

The conventional method for monitoring infection in the mouse model is based on the estimation of parasite loads in target organs by anatomopathological observations, such as microscope examination of lesions $[5,6]$. However, these techniques are cumbersome, laborious, and render the longitudinal monitoring of an infection in the same animal exceedingly difficult. To better investigate parasite expansion, rapid, nondestructive, real-time monitoring methods that are adaptable to the experimental animal model are needed. Over the past decade, noninvasive small animal imaging has gained increasing importance in research because it enables to study disease development with time in the same animal and often reduces 
the overall number of animals needed. The feasibility of utilizing in vivo imaging to monitor the infected murine models is becoming increasingly recognized. Fluorescence models, such as Salmonella [7] and bacterial meningitis [8], have been established but the development of noninvasive in vivo imaging techniques for helminth infection has not followed.

As far as we know, no in vivo fluorescent model for AE has been developed. We showed here that the JC-1, a cationic dye capable of exhibiting potential-dependent accumulation in mitochondria, can be used as a marker to constitute a realtime in vivo fluorescent imaging model for protoscolices from E. multilocularis. Metformin (Met), as an anti-malarial compound, has been proved to be effective against helminths, such as Brugia malayi [9], due to its inhibition of dihydrofolate reductase. Met is actively transported into hepatocytes [10] and thus achieves higher local concentration in liver which is the predominant lesion of AE than many conventional anti-helminthic chemical drugs. This prompted us to test whether it is also active against $E$. multilocularis. Using this model, we assessed the efficiency of Met against $E$. multilocularis protoscolices which could be enhanced when combined with ABZSO.

Male BALB/c mice (6-8 weeks old) were obtained from Xinjiang Medical University (Urumqi, China) and housed in specific-pathogen-free facilities for this study. E. multilocularis metacestodes were maintained in Mongolian gerbils (Meriones unguiculatus) by intraperitoneal injection of grinded metacestode tissue. After 6-8 weeks, the gerbils were euthanized with $\mathrm{CO}_{2}$. Metacestodes were isolated from the peritoneal cavity and cut into pieces. After being washed with PBS (Gibco/Invitrogen, Gaithersburg, Maryland, USA), these pieces were put into a tea strainer and strained through the sieve using the back end of a syringe plunger. Metacestodes were rinsed and then with $1 \times$ PBS to get the protoscolices [11]. Parasites $\left(\mathrm{n}=3 \times 10^{3} / 9.5-\mathrm{cm}^{2}\right.$ growth area per well) were routinely cultured at $37^{\circ} \mathrm{C}$ in RPMI-1640 medium supplemented with $10 \%$ fetal calf serum (Gibco/Invitrogen). Vitality was determined by the methylene blue exclusion test [12]. Test dosages for Met (Sigma, St. Louis, Missouri, USA) were 1, 5, and $10 \mathrm{mM}$ with the intent to find an optimal dosage. The test dosage for ABZSO (Hong Rui Kang Reagent Co, Wuhan, China) was $15 \mu \mathrm{M}$, a dosage which had been previously found to yield efficacy against E. multilocularis [13]. Each viability experiment was performed using 3 replicates per treatment condition and repeated 3 times. This study was approved by the Medical Ethics Committee of the Shihezi University, School of Medicine, Shi- hezi, China.

Control, $10 \mathrm{mM}$ Met-treated and $10 \mathrm{mM}$ Met combined with $15 \mu \mathrm{M}$ ABZSO-treated protoscolices were incubated with $10 \mathrm{mg} / \mathrm{ml} \mathrm{JC-1}$ dye dissolved in DMSO (Gibco/Invitrogen) for $30 \mathrm{~min}$ at room temperature. After incubation, parasites were washed with $20 \mathrm{mM}$ HEPES buffer (Gibco/Invitrogen), $\mathrm{pH}$ 7.2, and images were taken using a confocal microscope (LSM 800, Zeiss, Oberkochen, Germany). The intensities of red (excitation/emission wavelength $=485 / 590 \mathrm{~nm}$ ) and green (excitation/emission wavelength $=485 / 530$ ) fluorescence were analyzed for 20 individual protoscolices from control and treatedsamples. Images were analyzed using Image J software. The ratio of red to green fluorescence of JC-1 images was calculated using Stata 12.0.

Mice infected with JC-1 tagged parasites were imaged using an intensified charged coupled device camera of the in vivo imaging system (IVIS Lumina-IV) (Perkin Elmer, Waltham, Massachusetts, USA). To minimize background when imaging, the normal diet was replaced by a purified diet (GB14924, Trophic) during 7 days, since chlorophyll fluoresces naturally emitting between 675 and $685 \mathrm{~nm}$ and is detected in the 700 nm channel. Nevertheless, animals were fasted overnight, although water was allowed freely before acquiring the images in order to assure that feces were removed from intestines. The animals were shaved and the inbuilt spectral unmixing method of IVIS Lumina system was used to extract the signal of JC-1 from the tissue autofluorescence, which also eliminated the background signal due to residue fur. The animals were then lightly anesthetized with $5 \%$ chloral hydrate, placed in the camera chamber, and the fluorescence signal was acquired for $1 \mathrm{sec}$. Fluorescence determinations, recorded by the IVIS 100 system, were expressed as a pseudo color on a grey background, with yellow color denoting the highest intensity and dark red the lowest one.

Data within experiments were analyzed using Stata 12.0; significance was determined using the student's $t$-test and $P<0.05$ was considered statistically significant. All data are shown as the arithmetic mean \pm SEM.

The mortality percentage of protoscolices was analyzed in response to various Met concentrations. As can be seen in Fig. $1 \mathrm{~A}, 2$ days exposure led to a dose-dependent decrease in the viability of protoscolices. At $5 \mathrm{mM}$ Met, parasites revealed significant difference in vitality as compared with the control. The best results were obtained with Met where $72.57 \pm 5.71 \%$ of protoscolices were dead after 2 days when the dosage was 
$10 \mathrm{mM}$. Furthermore, the anti-echinococcosis activity of Met and its combination with ABZSO was tested against the isolated Met. An increased efficacy was found when a combination of Met plus ABZSO was used.

Aimed to test the possible inhibitory effect of Met on the parasite respiratory chain, we studied the mitochondrial func-
A

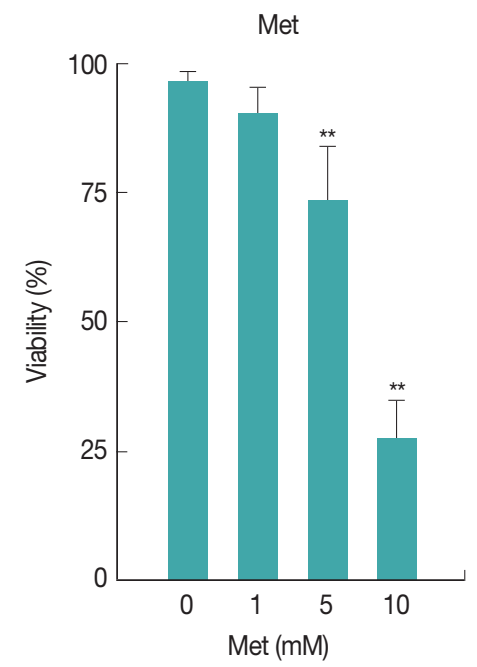

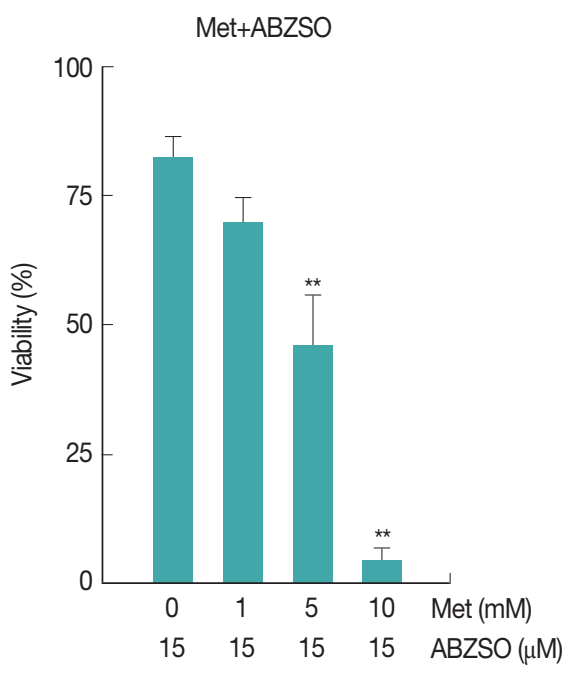

\section{B}

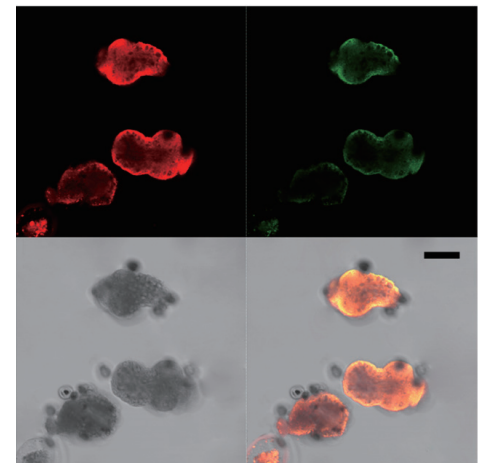

Con

\section{C}

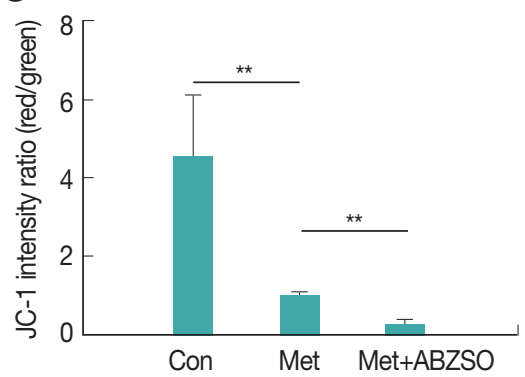

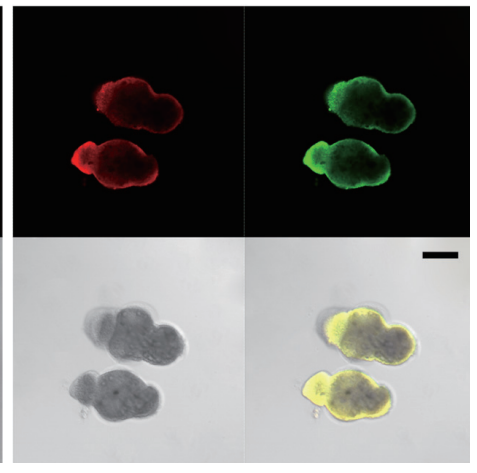

Met
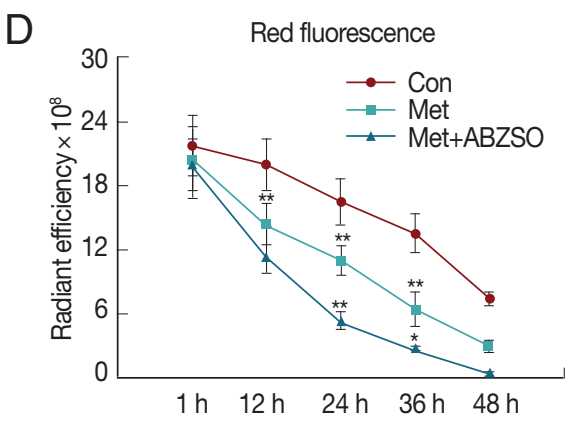

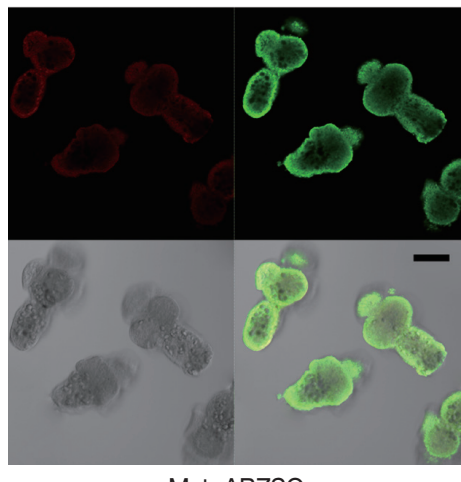

Met+ABZSO

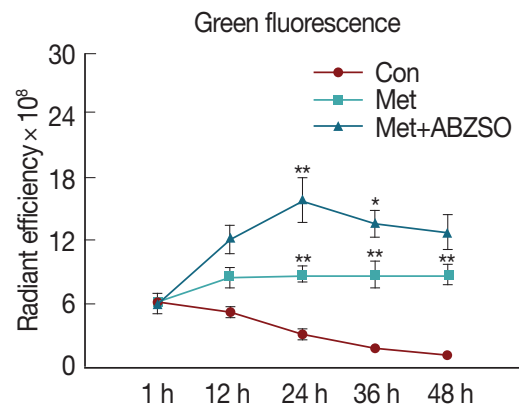

Fig. 1. Effect of Met and its combination with $A B Z S O$ on viability and mitochondrial function of protoscolices of E. multilocularis. (A) Viability of protoscolices incubated for 2 days, respective with varieties of Met and ABZSO concentration, in histograms. (B) Representative confocal images showing JC-1 fluorescence in protoscolices incubated under control conditions or treated with $10 \mathrm{mM}$ Met or $10 \mathrm{mM}$ Met combined with $15 \mu \mathrm{M}$ ABZSO for $12 \mathrm{hr}$ (scale bar indicates $100 \mu \mathrm{m}$ ). (C) Histograms showing the values of the red/green JC-1 fluorescence ratios measured in 3 groups of parasites by Image $\mathrm{J}$ Software. (D) Red and green fluorescence of 3 groups of protoscolices. The changing trend of fluorescence signal of control protoscolices (Con; $\bullet$ ), Met-treated protoscolices (Met; - ), and Met combined with ABZSO-treated protoscolices (Met+ABZSO; $\mathbf{\Lambda}$ ) as arbitrary units (AU) with the change of time. Experiments were carried out with $2 \times 10^{3}$ parasites by triplicate, and error bars represent SDs. Comparison of fluorescent intensity between any 2 groups of parasites at different time points was made. ${ }^{\star} P<0.05$; ${ }^{\star \star} P<0.01$; from 2-tails of Student's $t$-test. 
tional status using the membrane potential $(\Delta \Psi \mathrm{m})$ indicator JC-1. JC-1, a positively charged fluorescent compound [14], has been generally considered to be a reliable and sensitive fluorescent probe for detecting differences in $\Delta \Psi \mathrm{m}$ due to its dual emission characteristics. At high concentrations, JC-1 forms aggregates, known as J-aggregates, which emit red fluorescence. At low concentrations, this molecule exists mainly in a monomeric form which emits green fluorescence. Thus, since the amount of cationic dye taken up by the mitochondrion depends on its transmembrane potential, in normal mitochondria with high $\Delta \Psi \mathrm{m}$, the fluorescence emission will be mostly red. Whereas, in damaged mitochondria with low $\Delta \Psi \mathrm{m}$, it remains in the monomeric form, which exhibits only green fluorescence $[15,16]$.

JC-1 fluorescence of control, Met-treated, and Met com- bined with ABZSO-treated protoscolices was analyzed by confocal microscopy. Results of a typical experiment performed under these conditions are shown in Fig. 1B and C. Following $24 \mathrm{hr}$ treatment, untreated protoscolices showed a ratio of red to green fluorescence with a mean value of 4.5 , whereas Met treated protoscolices showed a lower mean ratio of around 1.1. Met combined with ABZSO treatment caused further reduction of the ratio to 0.29 . Met treatment induced destruction of protoscolices mitochondrial function as indicated by the vanishing of red fluorescence and an increase in green fluorescence. This efficacy can be enhanced by ABZSO.

Next, we tracked and monitored red and green fluorescence, respectively. As shown in Fig. 1D, red fluorescence of JC-1 in Met combined with ABZSO-treated protoscolices declined more rapidly as compared to that in Met-treated and control
A

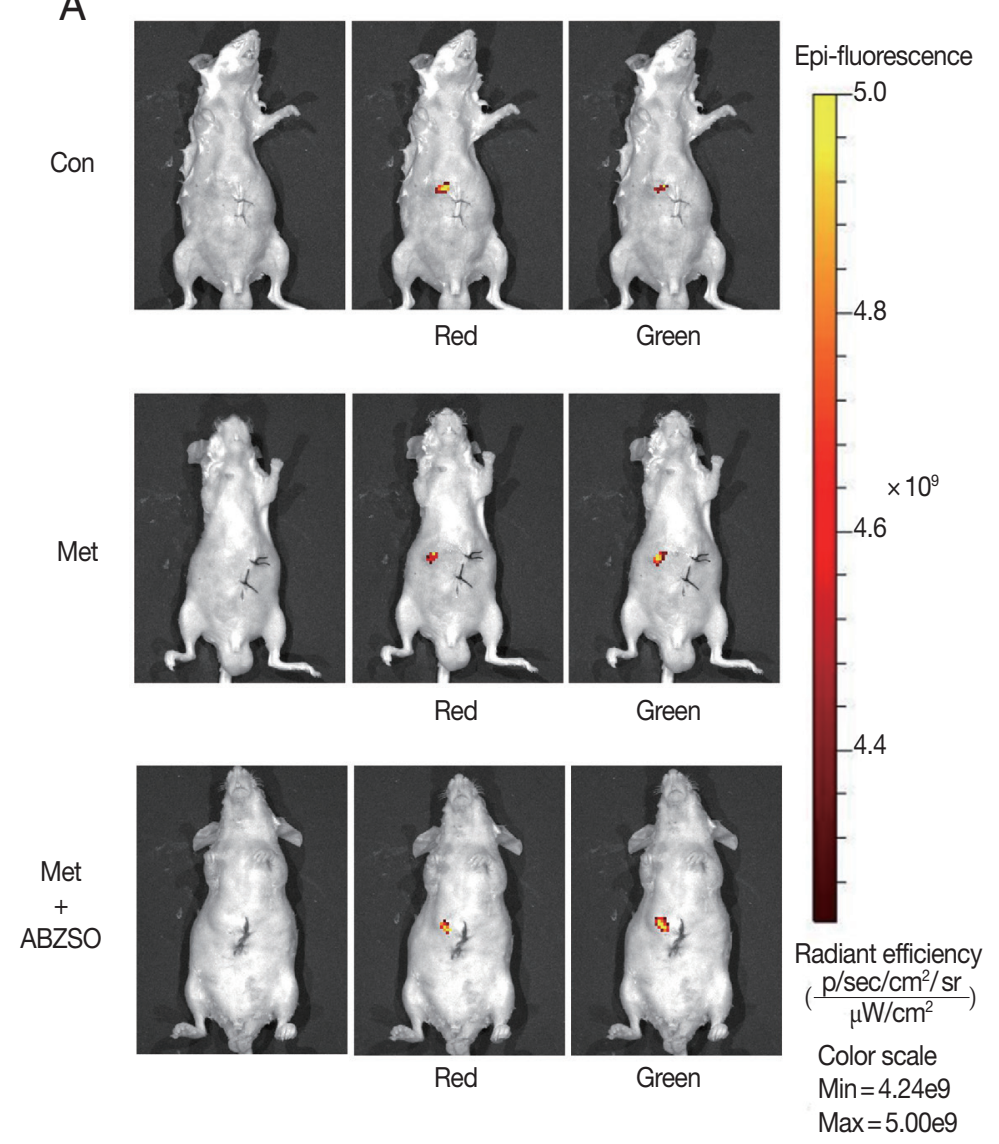

B

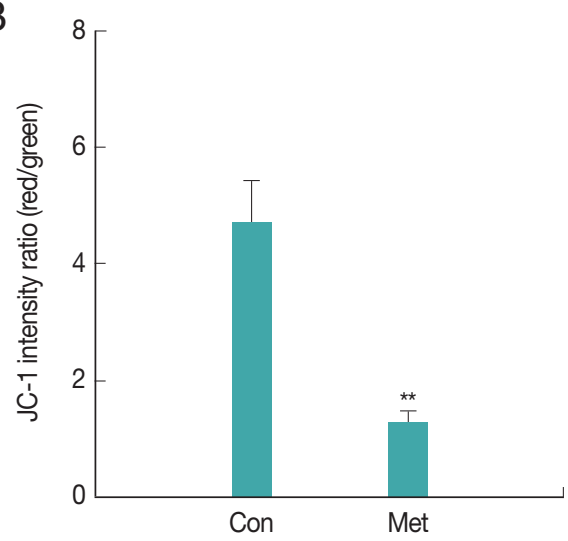

C

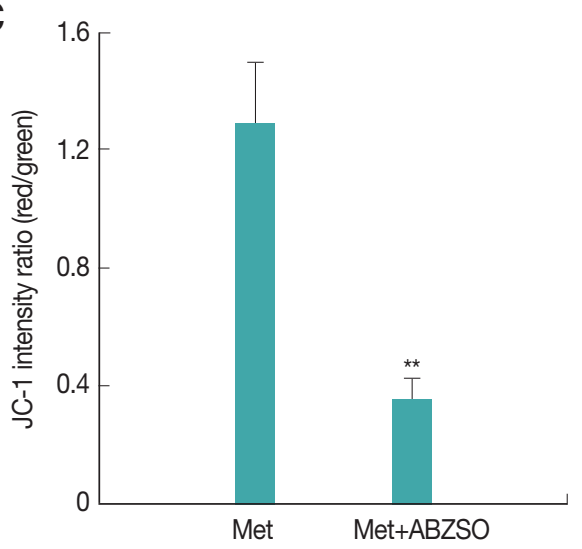

Fig. 2. Fluorescent imaging was performed on BALB/c mice $(n=10)$ challenged with $3 \times 10^{3}$ control protoscolices, Met-treated protoscolices or Met combined with ABZSO-treated protoscolices after $1 \mathrm{~min}$ of inoculation. JC-1 fluorescence was measured using an in vivo small-animal imaging system. (A) Representative images of individual mouse from each group. Quantified data represented radiant efficiency for parasites in $\mathrm{p} / \mathrm{sec} / \mathrm{cm}^{2} / \mathrm{sr} / \mu \mathrm{W} / \mathrm{cm}^{2}$. (B, C) Histograms showing the values of the red/green JC-1 fluorescence ratios measured in control, Met-treated protoscolices and Met combined with ABZSO treated protoscolices. ${ }^{* \star} P<0.01$; from 2-tails of Student's ttest. 
protoscolices. The fact that the red fluorescence of JC-1 in control parasites in this assay also decreased without adding Met or ABZSO was most probably due to the leakage and fluorescence quenching of the probe. For Met combined with ABZSO treatment, green fluorescence was evident at $12 \mathrm{hr}$ and peaked at $24 \mathrm{hr}$ before reducing. This indicated that the increase of green fluorescence caused by the decrease of viability of parasites was partly offset by fluorescence decay after $24 \mathrm{hr}$. Met group shared the same changing trend with it, but the trend of the latter was less significant. Green fluorescence of the control group decreased gradually over the whole experiment from 1 hr to $48 \mathrm{hr}$.

Next, the applicability of the fluorescent protoscolices for in vivo imaging in BALB/c mouse was tested. Three groups of mice were injected with Met-treated protoscolices, Met combined with ABZSO-treated protoscolices, and control protoscolices, respectively. Each animal received $2 \times 10^{3}$ parasites, and the inoculation was beneath the liver capsule. Hydrogel (Gibco/Invitrogen) was used to plug the pinhole, which prevented the protoscolices form leaking into the abdominal cavity. A bright and intense signal was detected after JC-1 tagged parasites injection allowing tracing the infection site.

As shown in Fig. 2, JC-1 fluorescent intensity ratio (red/ green) in the Met-treated protoscolices was significantly lower than that in controls. The ratio in Met combined with ABZSOtreated group was the lowest. This intensity proportional rela-
A

Red
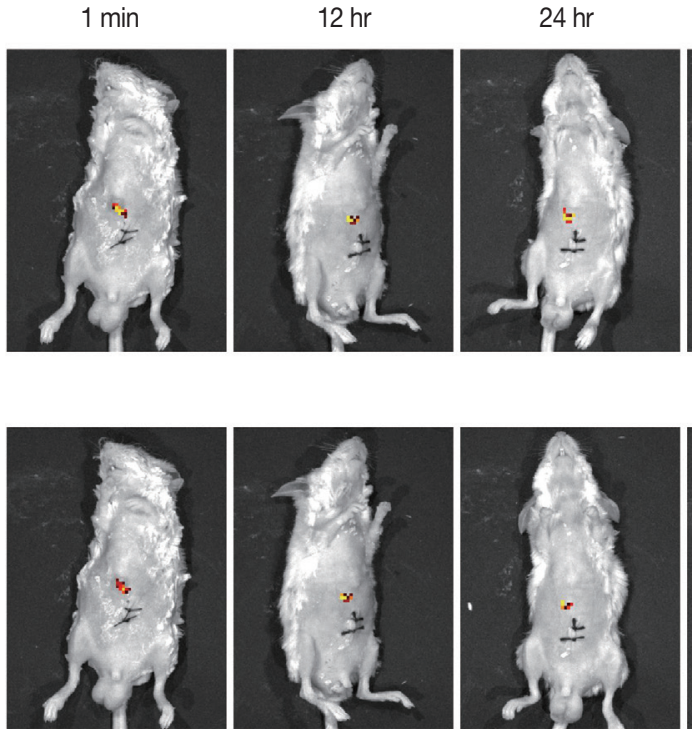

B
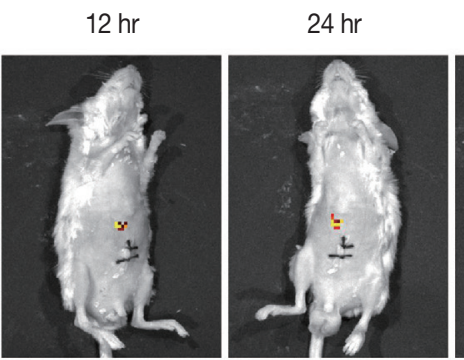

Green

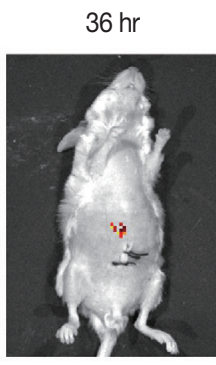

Epi-fluorescence

$\Gamma^{5.0}$

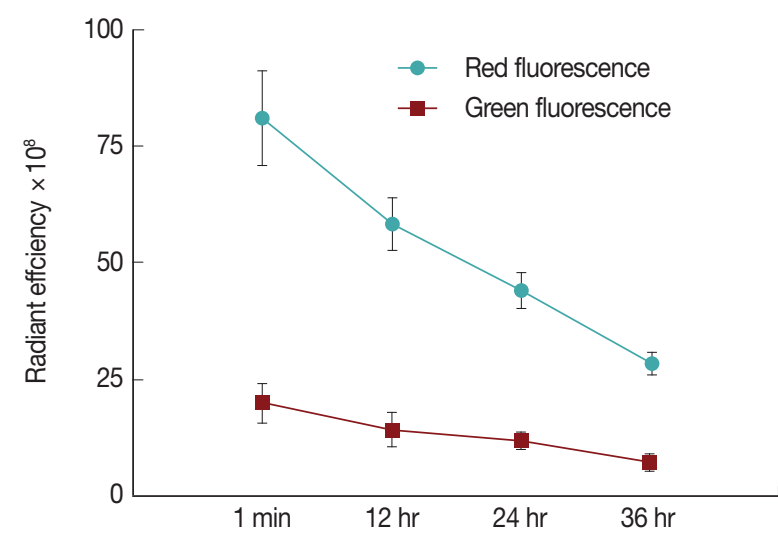

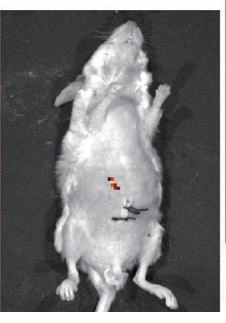

$-4.4$

$\times 10^{9}$

$-4.6$

Radiant efficiency $\left(\frac{\mathrm{p} / \mathrm{sec} / \mathrm{cm}^{2} / \mathrm{sr}}{\mu \mathrm{W} / \mathrm{cm}^{2}}\right)$

Color scale

$\mathrm{Min}=4.24 \mathrm{e} 9$

$\operatorname{Max}=5.00 \mathrm{e} 9$

Fig. 3. Fluorescent changing process in mice inoculated with untreated protoscolices. (A) Mice inoculated with untreated protoscolices as imaged to monitor the red and green fluorescence, respectively, at different time points after inoculation. (B) The changing trend of $\operatorname{Red}(\bullet)$ and Green $(\bullet)$ fluorescent intensity with the change of time in a line chart. Experiments were carried out with $3 \times 10^{3}$ parasites by triplicate, and error bars represent SDs. 
tionship was in consistent with the in vitro results. These data demonstrated that $\mathrm{AE}$ infections can be imaged in vivo by fluorescence. In other words, the locality and viability of protoscolices in living mice could be assessed by measuring the JC-1 fluorescent intensity ratio without the need to sacrifice the animals.

Finally, to explore the fluorescence changes over time, we monitored the in vivo fluorescence changing process in mice inoculated with untreated protoscolices. As shown in Fig. 3, red fluorescence was decayed faster than green fluorescence. This may be because the anti-photobleaching ability of monomeric form of JC-1 is stronger than that of the aggregate. Moreover, due to the low base intensity, the green fluorescence could hardly be imaged after $36 \mathrm{hr}$. These results suggest that the fluorescent protoscolices can be tracked in vivo for about $36 \mathrm{hr}$ before the fluorescence disappears.

Understanding E. multilocularis infection in the murine models with fine temporal resolution requires the development of imaging strategies that can reveal cellular and molecular changes in the living organism. In the present study, we aimed to assess the feasibility of using in vivo fluorescent imaging as a quantitative tool to assess the load of parasites. Here, we detected and monitored fluorescent parasites after the course of Met treatment and Met combined with ABZSO treatment using JC-1 as a marker. We showed that JC-1 can be used to establish in vivo fluorescent model of AE infection. In addition, using confocal microscopy and this model, we confirmed that the anti-malarial compound Met exhibits promising activity against $E$. multilocularis protoscolices in vitro. Biguanide is a well known anti-malarial drug which has been in clinical use for more than 50 years [17]. Antimalarial drugs were shown to be active against a broad spectrum of other parasitic diseases, such as trypanosomiasis, leishmaniasis, and amebiasis [18-20]. The conventional view is that biguanide is metabolically converted to the cyclic metabolite cycloguanil, a potent inhibitor of parasite dihydrofolate reducatase. This inhibition may be associated with reductions in mitochondrial functions, resulting in cellular damage and death [21].

In the past decades, researchers have routinely relied on microscopy and quantitative PCR with tissue homogenates to determine parasite burdens in organs of interest [22]. While useful, these methods have several drawbacks. First, the animal analyzed must be sacrificed, which makes it impossible for researchers to monitor the extent and kinetics of disease progression in the same animal with the change of time. This means that the significant individual differences between animals may be missed. In addition, many more animals must be used to obtain convincing data when multiple time points need to be examined. Second, the traditional methods are complicated and rely on knowing which tissue to examine; diffuseness to unexpected anatomical sites or even to particular tissues of the organ being examined may easily be missed. The noninvasive characteristic of fluorescent imaging surmounts many of these problems. Procedures can be performed repeatedly, which allows each animal to be used as its own control [23]. Averaging effects, therefore, are eliminated at the same time that the total number of animals required is greatly reduced. The noninvasive approach we described here is significantly more rapid and convenient than conventional assays.

In vivo fluorescent imaging model has the potential to promote research into $\mathrm{AE}$ and other parasitic infections, but this model will probably not completely substitute for traditional methods. The efficacy of fluorescence passage across tissues can be influenced by various factors, including organ localization, tissue density, and depth of the fluorescence emitter [24]. Detection of parasites is limited when low parasite number is to be analyzed in sites covered by dense structures. Thus, levels of fluorescent emissions may vary between organs and quantification of parasite numbers in vivo requires the generation of organ-specific algorithms to validate the comparison of lesions in different organs.

In conclusion, our study is the first to use JC-1 successfully to provide in vivo fluorescent imaging in a murine model of AE. Although this in vivo imaging model is transient rather than stable and thus cannot meet the demand of long term monitoring, and although it requires the further development of genetic manipulation of helminth $[25,26]$ to introduce a certain reporter gene, it could prove to be of significant value in future in vivo chemical drug trials against $E$. multilocularis when some long term fluorescent markers are acquired. Work toward these aims is currently underway.

\section{ACKNOWLEDGMENT}

This study was supported by Basic Science Research Program through the National Natural Science Foundation of China (grant nos. 81260412, 81360453).

\section{CONFLICT OF INTEREST}

We have no conflict of interest related to this work. 


\section{REFERENCES}

1. Giraudoux P, Raoul F, Afonso E, Ziadinov I, Yang Y, Li L, Li T, Quere JP, Feng X, Wang Q, Wen H, Ito A, Craig PS. Transmission ecosystems of Echinococcus multilocularis in China and Central Asia. Parasitology 2013; 140: 1655-1666.

2. Gottstein B, Stojkovic M, Vuitton DA, Millon L, Marcinkute A, Deplazes P. Threat of alveolar echinococcosis to public health - a challenge for Europe. Trends Parasitol 2015; 31: 407-411.

3. Eckert J, Deplazes P. Biological, epidemiological, and clinical aspects of echinococcosis, a zoonosis of increasing concern. Clin Microbiol Rev 2004; 17: 107-135.

4. Hemphill A, Stadelmann B, Rufener R, Spiliotis M, Boubaker G, Muller J, Muller N, Gorgas D, Gottstein B. Treatment of echinococcosis: albendazole and mebendazole--what else? Parasite 2014; 21: 70.

5. Kuster T, Stadelmann B, Hermann C, Scholl S, Keiser J, Hemphill $\mathrm{A}$. In vitro and in vivo efficacies of mefloquine-based treatment against alveolar echinococcosis. Antimicrob Agents Chemother 2011; 55: 713-721.

6. Emery I, Bories C, Liance M, Houin R. In vitro quantitative assessment of Echinococcus multilocularis metacestode viability after in vivo and in vitro main-tenance. Int J Parasitol 1995; 25: 275278.

7. Contag CH, Contag PR, Mullins JI, Spilman SD, Stevenson DK, Benaron DA. Photonic detection of bacterial pathogens in living hosts. Mol Microbiol 1995; 18: 593-603.

8. Kadurugamuwa JL, Modi K, Yu J, Francis KP, Orihuela C, Tuomanen E, Purchio AF, Contag PR. Noninvasive monitoring of pneumococcal meningitis and evaluation of treatment efficacy in an experimental mouse model. Mol Imaging 2005; 4: 137142.

9. Bag S, Tawari NR, Sharma R, Goswami K, Reddy MV, Degani MS. In vitro biological evaluation of biguanides and dihydrotriazines against Brugia malayi and folate reversal studies. Acta Trop 2010; 113: 48-51.

10. Wilcock C, Bailey CJ. Accumulation of metformin by tissues of the normal and diabetic mouse. Xenobiotica 1994; 24: 49-57.

11. Reuter S, Merkle M, Brehm K, Kern P, Manfras B. Effect of amphotericin B on larval growth of Echinococcus multilocularis. Antimicrob Agents Chemother 2003; 47: 620-625.

12. Ceballos L, Elissondo C, Sanchez Bruni S, Denegri G, Lanusse C, Alvarez L. Comparative performances of flubendazole and albendazole in cystic echinococcosis: ex vivo activity, plasma/cyst disposition, and efficacy in infected mice. Antimicrob Agents Chemother 2011; 55: 5861-5867.
13. Ingold K, Bigler P, Thormann W, Cavaliero T, Gottstein B, Hemphill A. Efficacies of albendazole sulfoxide and albendazole sulfone against in vitro cultivated Echinococcus multilocularis metacestodes. Antimicrob Agents Chemother 1999; 43: 10521061.

14. Reers M, Smith TW, Chen LB. J-aggregate formation of a carbocyanine as a quantitative fluorescent indicator of membrane potential. Biochemistry 1991; 30: 4480-4486.

15. Pan H, Cheng L, Yang H, Zou W, Cheng R, Hu T. Lysophosphatidic acid rescues human dental pulp cells from ischemia-induced apoptosis. J Endod 2014; 40: 217-222.

16. Bakstad D, Adamson A, Spiller DG, White MR. Quantitative measurement of single cell dynamics. Curr Opin Biotechnol 2012; 23: 103-109.

17. Jones K, Ward SA. Biguanide-atovaquone synergy against Plasmodium falciparum in vitro. Antimicrob Agents Chemother 2002; 46: 2700-2703.

18. Barrett MP. Problems for the chemotherapy of human African trypanosomiasis. Curr Opin Infect Dis 2000; 13: 647-651.

19. Copeland NK, Aronson NE. Leishmaniasis: treatment updates and clinical practice guidelines review. Curr Opin Infect Dis 2015; 28: 426-437.

20. Gupta YK, Gupta M, Aneja S, Kohli K. Current drug therapy of protozoal diarrhoea. Indian J Pediatr 2004; 71: 55-58.

21. Srivastava IK, Vaidya AB. A mechanism for the synergistic antimalarial action of atovaquone and proguanil. Antimicrob Agents Chemother 1999; 43: 1334-1339.

22. Gottstein B, Hemphill A. Echinococcus multilocularis: the parasitehost interplay. Exp Parasitol 2008; 119: 447-452.

23. Tiernan JP, Ingram N, Marston G, Perry SL, Rushworth JV, Coletta PL, Millner PA, Jayne DG, Hughes TA. CEA-targeted nanoparticles allow specific in vivo fluorescent imaging of colorectal cancer models. Nanomedicine 2015; 10: 1223-1231.

24. Calvo-Alvarez E, Stamatakis K, Punzon C, Alvarez-Velilla R, Tejeria A, Escudero-Martinez JM, Perez-Pertejo Y, Fresno M, BalanaFouce R, Reguera RM. Infrared fluorescent imaging as a potent tool for in vitro, ex vivo and in vivo models of visceral leishmaniasis. PLoS Negl Trop Dis 2015; 9: e0003666.

25. Spiliotis M, Lechner S, Tappe D, Scheller C, Krohne G, Brehm K. Transient transfection of Echinococcus multilocularis primary cells and complete in vitro regeneration of metacestode vesicles. Int J Parasitol 2008; 38: 1025-1039.

26. Mizukami C, Spiliotis M, Gottstein B, Yagi K, Katakura K, Oku Y. Gene silencing in Echinococcus multilocularis protoscolices using RNA interference. Parasitol Int 2010; 59: 647-652. 